\title{
Nucleosome repositioning links DNA (de)methylation and differential CTCF binding during stem cell development
}

\author{
Vladimir B. Teif, ${ }^{1}$ Daria A. Beshnova, ${ }^{1}$ Yevhen Vainshtein, ${ }^{2}$ Caroline Marth, ${ }^{1}$ \\ Jan-Philipp Mallm, ${ }^{1}$ Thomas Höfer, $^{2}$ and Karsten Rippe ${ }^{1}$ \\ ${ }^{1}$ Research Group Genome Organization and Function, Deutsches Krebsforschungszentrum (DKFZ) and BioQuant, 69120 Heidelberg, \\ Germany; ${ }^{2}$ Division Theoretical Systems Biology, Deutsches Krebsforschungszentrum (DKFZ) and BioQuant, 69120 Heidelberg, \\ Germany
}

\begin{abstract}
During differentiation of embryonic stem cells, chromatin reorganizes to establish cell type-specific expression programs. Here, we have dissected the linkages between DNA methylation $(5 \mathrm{mC})$, hydroxymethylation $(5 \mathrm{hmC})$, nucleosome repositioning, and binding of the transcription factor CTCF during this process. By integrating MNase-seq and ChIP-seq experiments in mouse embryonic stem cells (ESC) and their differentiated counterparts with biophysical modeling, we found that the interplay between these factors depends on their genomic context. The mostly unmethylated $\mathrm{CpG}$ islands have reduced nucleosome occupancy and are enriched in cell type-independent binding sites for CTCF. The few remaining methylated $\mathrm{CpG}$ dinucleotides are preferentially associated with nucleosomes. In contrast, outside of $\mathrm{CpG}$ islands most $\mathrm{CpGs}$ are methylated, and the average methylation density oscillates so that it is highest in the linker region between nucleosomes. Outside CpG islands, binding of TET1, an enzyme that converts $5 \mathrm{mC}$ to $5 \mathrm{hmC}$, is associated with labile, MNase-sensitive nucleosomes. Such nucleosomes are poised for eviction in ESCs and become stably bound in differentiated cells where the TET1 and $5 \mathrm{hmC}$ levels go down. This process regulates a class of CTCF binding sites outside $\mathrm{CpG}$ islands that are occupied by CTCF in ESCs but lose the protein during differentiation. We rationalize this cell type-dependent targeting of CTCF with a quantitative biophysical model of competitive binding with the histone octamer, depending on the TET1, 5hmC, and $5 \mathrm{mC}$ state.
\end{abstract}

[Supplemental material is available for this article.]

DNA cytosine modifications such as methylation $(5 \mathrm{mC})$ and hydroxymethylation $(5 \mathrm{hmC})$ are important determinants of cell differentiation (Reddington et al. 2013; Smith and Meissner 2013). The site-specific locations of these DNA modifications have been linked to disease development through nucleosome positioning (Portela et al. 2013), transcription factor (TF) binding (Stadler et al. 2011), and differential gene expression (Reddington et al. 2013; Smith and Meissner 2013). For the relation of $5 \mathrm{mC}$ positions and genomic nucleosome arrangement, conflicting conclusions have been reported since this question was first addressed in the 1970s (Razin and Cedar 1977). Recent studies using high-throughput sequencing have found $5 \mathrm{mCs}$ to reside preferentially either inside nucleosomes (Chodavarapu et al. 2010; Li et al. 2012; Collings et al. 2013) or in the linker DNA regions between nucleosomes (Felle et al. 2011; Kelly et al. 2012). In addition, it is an open question as to how cytosine hydroxymethylation (5hmC) (Williams et al. 2011; Xu et al. 2011; Spruijt et al. 2013; Sun et al. 2013) and formylation (5fC) (Raiber et al. 2012; Shen et al. 2013; Song et al. 2013) relate to nucleosome positions. Furthermore, DNA methylation and nucleosome positioning appear to be linked with TF binding and gene expression in a complex manner. For some proteins that can specifically recognize either methylated (Baubec et al. 2013) or unmethylated CpG DNA dinucleotides (Thomson et al. 2010), a direct link between the DNA methylation status and binding is

Corresponding authors: V.Teif@dkfz.de, Karsten.Rippe@dkfz.de Article published online before print. Article, supplemental material, and publication date are at http://www.genome.org/cgi/doi/10.1101/gr.164418.113. well established. Many other TFs do not contain a dedicated CpG recognition domain, but CpGs are present in their DNA consensus motifs, which can lead to DNA methylation-dependent binding (Hu et al. 2013b). The majority of TF consensus sequence motifs (not necessarily bound by TFs) are hypermethylated in vivo (Choy et al. 2010), and demethylation of these sites can promote TF binding (Wiench et al. 2011). Alternatively, TF binding could be an upstream, methylation-independent event responsible for recruiting enzymes, which then change the methylation state (Stadler et al. 2011). An exemplary case for the complex interplay between DNA methylation and TF binding is the transcription factor CTCF. It recognizes its DNA target via a highly conserved zinc finger protein domain. CTCF demarcates boundaries between chromatin domains and is involved in the regulation of gene expression via establishing specific 3D chromatin structures through DNA loop formation (Merkenschlager and Odom 2013; Van Bortle and Corces 2013). About $40 \%$ of the several thousands of potential cell typespecific CTCF binding sites in the human genome were linked to changes in DNA methylation (Wang et al. 2012). On the other hand, it was concluded that in most cases, differential DNA methylation is not a cause but rather a consequence of CTCF binding (Stadler et al. 2011; Feldmann et al. 2013). Recent reports showed that changes of CTCF binding during cell differentiation occur

\footnotetext{
(c) 2014 Teif et al. This article is distributed exclusively by Cold Spring Harbor Laboratory Press for the first six months after the full-issue publication date (see http://genome.cshlp.org/site/misc/terms.xhtml). After six months, it is available under a Creative Commons License (Attribution-NonCommercial 4.0 International), as described at http://creativecommons.org/licenses/by-nc/4.0/.
} 
mostly at weaker CTCF sites (Plasschaert et al. 2013) and lead to functionally important variations in the chromatin states (Kasowski et al. 2013). However, it remains unclear what drives the selection of sites within the many weak CTCF motifs throughout the genome.

Here, we set out to dissect how nucleosome positioning, DNA (de)methylation and cell type-specific binding of CTCF are linked. To this end, we conducted MNase-seq experiments in mouse embryonic stem cells (ESCs), differentiated mouse neural progenitor cells (NPCs), and embryonic fibroblasts (MEFs). We applied a novel procedure of mapping nucleosome occupancy at three different levels of MNase digestion, yielding an average DNA fragment length from $\sim 180$ to $\sim 150 \mathrm{bp}$. Nucleosome maps detected at low levels of MNase digestion contain a higher fraction of labile nucleosomes that are lost upon moderate and high MNase digestion. In this manner, we obtained not only static regions of nucleosome depletion and enrichment as done previously (Teif et al. 2012), but were able to identify labile nucleosomes and correlate their positions with published high-resolution data with respect to DNA methylation (Stadler et al. 2011), CTCF binding (Shen et al. 2012), TET1 enrichment (Williams et al. 2011), hydroxymethylation (Yu et al. 2012), and formylation (Raiber et al. 2012), as well as the pattern of H3K4me3 and H3K9me3 histone modifications also determined in this study. Based on the experimental data and associated bioinformatical analysis, we developed a biophysical model, which allowed us to rationalize the $5 \mathrm{mC} /$ 5hmC-dependent competition for DNA-binding between CTCF and the histone octamer during stem cell development.

\section{Results}

DNA methylation in relation to nucleosomes depends on the chromatin context

To elucidate the $5 \mathrm{mC}$ distribution with respect to nucleosomes, we calculated the occurrence of methylated and unmethylated CpGs along each of the nucleosomal DNA fragments that we determined in mouse ESCs using paired-end sequencing (Fig. 1). CpGs were classified as being in- or outside of a CpG cluster using the algorithm of Hackenberg et al. (2006), which reports 125,000 CpG clusters or CpG islands (CGIs) for the mouse genome. We obtained qualitatively similar results when CpGs were classified into being in- or outside of CpG clusters as defined by the algorithm of Wu et al. (2010), which reports 75,000 CGIs for the mouse genome (data not shown). The 16,000 "classical" CGIs included in the UCSC Genome Browser for the mouse $\mathrm{mm} 9$ assembly would mostly appear as a subset of the $\mathrm{CpG}$ clusters defined above.

Figure 1A shows the average pattern of the distribution of methylated and unmethylated CpGs along the nucleosome, calculated for all genomic nucleosomes, which are mostly located outside of CGIs. DNA methylation was minimal at the middle of the nucleosome (close to the nucleosome dyad), and smoothly increased toward the ends of the nucleosomes up to the maximum methylation level between nucleosomes. This pattern holds true for both low and high levels of chromatin digestion with MNase (black and blue curves in Fig. 1A). Furthermore, the same pattern was confirmed by mapping the CpG occurrence along the dinucleosome using a purified dinucleosome chromatin fraction obtained by moderate MNase digestion, where the linker between two nucleosomes remained intact (Fig. 1B). The $5 \mathrm{mC}$ pattern followed the total CpG content, since most CpGs are methylated (Supplemental Fig. S1). This suggests that the relation of nucleosome positioning and DNA methylation is to some extent hard-wired in the DNA sequence. Both mono- and dinucleosome fractions were characterized by strong
A

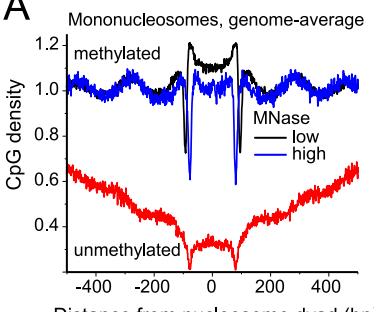

B

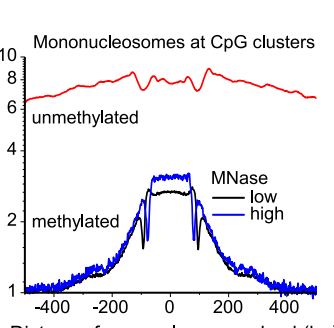

Distance from nucleosome dyad (bp)

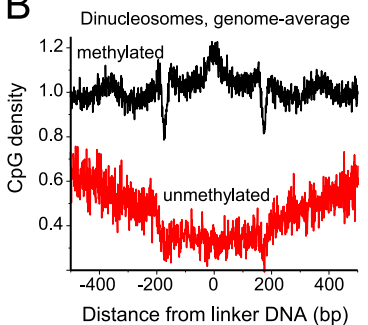

D

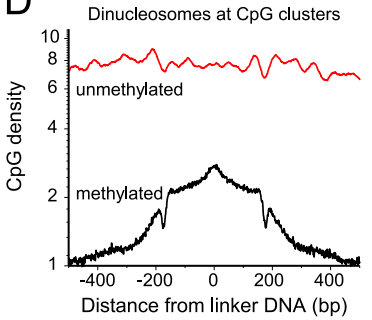

Figure 1. DNA methylation patterns relative to nucleosomes. $(A)$ Average $\mathrm{CpG}$ density of methylated $(>50 \% 5 \mathrm{mC})$ and unmethylated $(<10 \%$ $5 \mathrm{mC}$ ) mononucleosome sequences in ESCs for the complete genome. Most of the sequences reside outside of CGls because the latter represent only a fraction of $\sim 1 \%$ of the total pool of sequences mapped by MNase-seq. (Black line) DNA methylation along the nucleosomes obtained at low MNase digestion; (blue line) high MNase digestion. The nucleosome dyad is the middle of the nucleosomal DNA fragment. (B) Same as in panel $A$ but for the dinucleosome fraction. The linker is defined as the middle of the dinucleosome. (C) CpG density for mononucleosomes and associated linker DNA inside CGls. Putative CGls were taken from the coordinates of $125,303 \mathrm{CpG}$ clusters defined by the proximity of neighboring $\mathrm{CpG}$ with the CpGcluster2 algorithm (Hackenberg et al. 2006). (D) Same as in panel C but for the dinucleosome fraction.

oscillations of $5 \mathrm{mCs}$ with the period equal to the nucleosome repeat length. These $5 \mathrm{mC}$ oscillations were in counter-phase with the nucleosome density (Supplemental Fig. S1C). The borders of both the nucleosome and dinucleosome DNA fragments were characterized by sharp dips in the $5 \mathrm{mC}$ density, which is likely to reflect the AT preference of MNase digestion (Fig. 1A,B). However, in the region that flanked the isolated DNA fragment, the $5 \mathrm{mC}$ oscillations persisted and thus represent the unperturbed genomic distribution (Fig. 1A). Furthermore, in dinucleosomes, the DNA linker in the middle between two nucleosomes had an $\sim 20 \%$ increased DNA methylation level (Fig. 1B). Since this linker region remained intact during dinucleosome DNA extraction, the $5 \mathrm{mC}$ enrichment in this region cannot be a result of MNase sequence preferences.

The characteristic oscillating DNA methylation pattern appeared to be specific for nucleosomes outside CGIs (Supplemental Fig. S1D). Inside CGIs, the opposite trend was observed (Fig. 1C,D). The sparsely methylated CpGs in these regions were preferentially associated with nucleosomes with a flat average DNA methylation profile. Thus, the local nucleosome $/ 5 \mathrm{mC}$ distribution was dependent on the genomic context, e.g., inside CGIs or not, which resolves the seeming controversy that existed in the literature with respect to this issue (Chodavarapu et al. 2010; Felle et al. 2011; Kelly et al. 2012; Li et al. 2012; Collings et al. 2013).

\section{Occupancy of CTCF binding sites within ESCs is determined by DNA sequence}

Having identified the basic features of the interplay between nucleosomes and DNA methylation, we set out to dissect how these

\section{Genome Research www.genome.org}


affect the binding of the CTCF transcription factor in ESCs. The $\sim 40,000$ sites bound by CTCF in ESCs were classified according to Stadler et al. into those within fully methylated regions (FMRs, characteristic for random genomic locations far from enhancers or promoters), low-methylated regions (LMRs, characteristic for enhancers), and unmethylated regions (UMRs, characteristic for active promoters) (Stadler et al. 2011). Figure 2A shows large differences in nucleosome density for large-scale clusters of DNA methylation. FMRs displayed a genome-average nucleosome density, LMRs had an $\sim 30 \%$ lower nucleosome density, and UMRs were nucleosomedepleted by $\sim 60 \%$. We then calculated average CTCF enrichment profiles for these three regions based on ChIP-seq data from the Mouse ENCODE Project (Shen et al. 2012). CTCF occupancies were about average in FMR regions, but were elevated in LMRs (10-fold CTCF enrichment) and UMRs (15-fold CTCF enrichment) (Fig. 2B). To test whether CTCF binding in these regions was determined by nucleosome occupancy and/or DNA methylation, we calculated DNA sequence-specific CTCF occupancy profiles for each of the several thousands of regions identified as FMR, LMR, and UMR with our previously introduced TFnuc software (Methods; Teif and Rippe 2012; Teif et al. 2013). TFnuc allows the studying of different scenarios of TF binding, taking as input protein concentrations, position-specific weight matrices (PWM), and nucleosome occupancy profiles. Following previous work (Goh et al. 2010), the CTCF binding constant was corrected by a factor of $1.5^{- \text {nuc_occup }}$, with nuc_occup being the experimentally determined nucleosome occupancy. The effect of DNA methylation on the binding constant was quantified in the same manner using the experimental $5 \mathrm{mC}$ density averaged in a 200-bp window in ESCs (Stadler et al. 2011). The CTCF binding occupancy as calculated from the PWM without accounting for nucleosomes and DNA methylation (Fig. 2C) predicted the experimental CTCF binding profiles at FMRs, LMRs, and UMRs (Fig. 2B). Since FMR regions are rather large (on average $\sim 50 \mathrm{~kb}$ ), there is no distinct peak of CTCF occupancy in the middle of FMRs, although these comprise many strong CTCF sites. Three different models were compared based on their corresponding receiver-operator curves (ROC), with the quality of the model being reflected by the area under the curve (AUC). One model takes into account only the PWM, while in the two others, the PWM was combined either with DNA methylation or with nucleosome occupancy (Fig. 2D). In the latter models, we assumed a linear effect of DNA methylation and nucleosome occupancy on the CTCF binding constant predicted from the PWM (see Methods). The predicted CTCF binding from the pure PWM model that considered only the DNA sequence was very good (AUC = 0.89) and confirmed the expectation that the ChIP-seq peak height represents binding site occupancy. Neither the introduction of DNA methylation, nor including the presence of nucleosomes significantly improved the predictions, with AUC $=0.90$ in both cases.

\section{DNA methylation alone is a poor predictor of differential CTCF binding between ESCs and differentiated cells}

A significant change of the global CTCF binding profile is observed when comparing ESCs, neural progenitor cells, and mouse embryonic fibroblasts. Since these cells have identical genomes, CTCF binding differences cannot be explained by the DNA sequence. Can they be explained by cell type-specific differences in DNA methylation as was previously proposed (Wang et al. 2012)? To answer this question, we compared three groups of CTCF sites classified according to the peak calling performed by the Mouse ENCODE
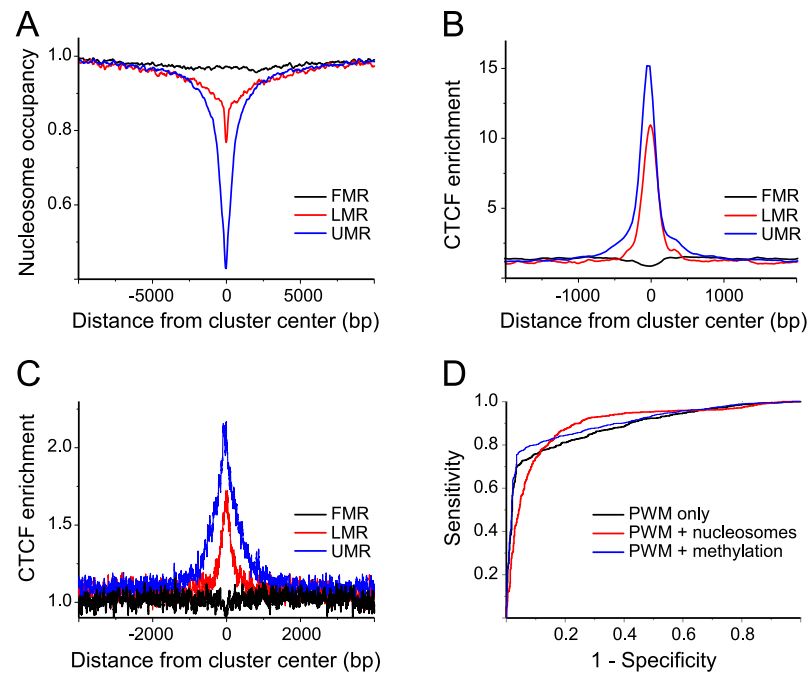

Figure 2. CTCF binding in ESCs can be explained solely by the DNA sequence. $(A)$ Average nucleosome occupancy profiles for genomic regions with different levels of DNA methylation. For fully methylated regions (FMR), the occupancy remains flat as compared to genome-average levels. Low methylated regions (LMR) and unmethylated regions (UMR) were nucleosome-depleted by $\sim 30 \%$ and $\sim 60 \%$, respectively. (B) Average CTCF enrichment calculated from ChIP-seq data for the three different classes of $5 \mathrm{mC}$ density. (C) Average CTCF enrichment predicted for the same regions based on the DNA sequence preferences given by the TRANSFAC PWM, without taking into account nucleosomes and DNA methylation. (D) Receiver operator curves calculated for the TFnuc model, taking into account only the CTCF weight matrix without nucleosomes and DNA methylation (black). In addition to PWM, competition with nucleosomes (red) or DNA methylation (blue) was considered. The area under the curve (AUC) reflects both the sensitivity and specificity and thus determines the goodness of the model.

Consortium (Shen et al. 2012) (see Methods): constitutive sites that were occupied by CTCF both in ESCs and MEFs (termed "ESC and MEF"), variable sites where CTCF occupancy in MEFs decreased at least twofold in comparison with ESCs ("ESC not MEF"), and a class of relatively weak sites for which ChIP-seq peaks were identified only in MEFs ("MEF not ESC"). In a subsequent analysis of the raw ChIP-seq data in ESCs or in MEFs, we detected essentially all ChIPseq peaks in both data sets. However, the peak heights were different for a large set of binding sites between ESCs and MEFs. Thus, CTCF binding had significant differences in occupancy between cell types but not in an all-or-none manner. The "ESC and MEF" occupancy data were normalized to unity (100\%) for the constitutively occupied sites as defined by sites that were present in both ESCs and MEFs, with an occupancy of $\sim 50$-fold above the background within a given cell type. The "ESC not MEF" sites comprised those sites for which the average occupancy decreased from $75 \%$ in ESCs to $20 \%$ in MEFs. The "MEF not ESC" class showed only 5\% change of occupancy, from $\sim 15 \%$ occupancy in MEFs to $\sim 10 \%$ occupancy in ESCs, and represents sites which are weak in both ESCs and in MEFs. Thus, the most significant changes of CTCF binding during ESC to MEF differentiation originated from a loss of CTCF at a fraction of the ESC binding sites, i.e., the "ESC not MEF" class. To rule out the possibility that the "MEF not ESC" and "ESC not MEF" sites are falsepositives of the ChIP-seq experiment, we compared these data sets with the recently determined ChIA-PET coordinates of CTCFmediated chromatin loops in ESCs (Handoko et al. 2011). We found that a similar percentage of $\sim 50 \%$ of sites from the ChIP-seq data sets overlapped with the ChIA-PET data. 


\section{A}

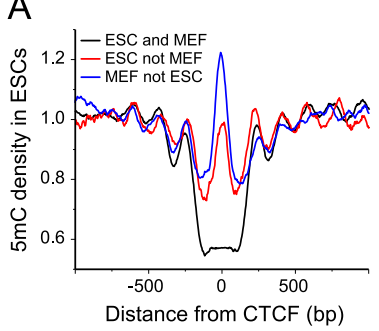

$\mathrm{B}$
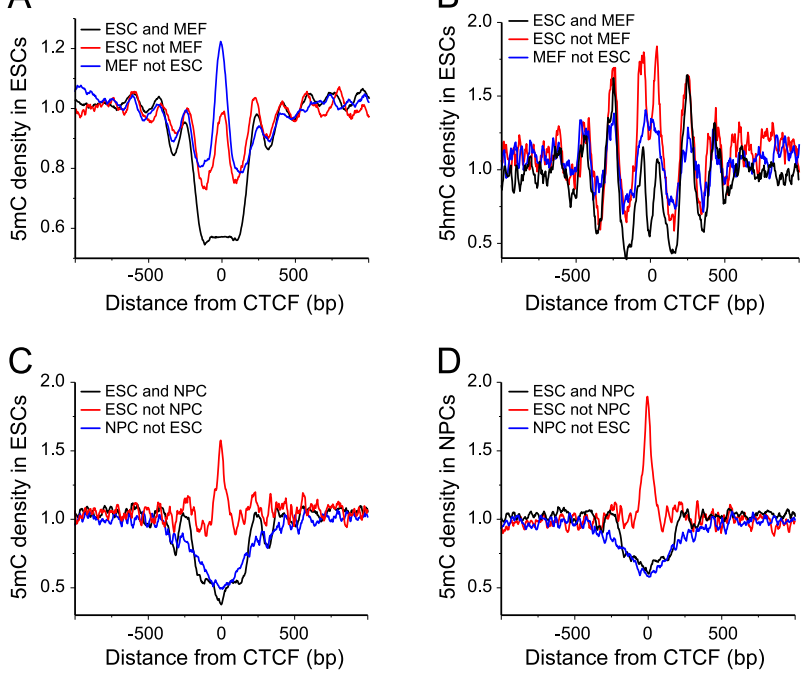

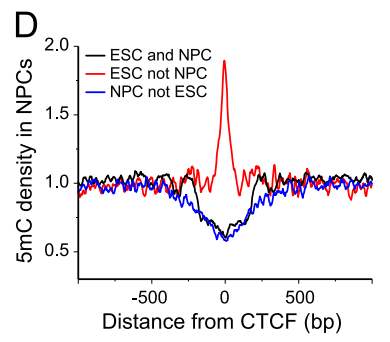

Figure 3. Distribution of $5 \mathrm{mC}$ and $5 \mathrm{hmC}$ around CTCF sites in ESCs, NPCs, and MEFs. (A) $5 \mathrm{mC}$ density calculated around constitutive ("ESC and MEF"), variable ("ESC not MEF"), and weak ("MEF not ESC") CTCF sites in ESCs from the published CTCF ChIP-seq data (Shen et al. 2012). (B) Same as panel $A$ but for the hydroxymethylation modification at CpGs. (C) $5 \mathrm{mC}$ density in ESCs at "ESC and NPC," "ESC not NPC" and "NPC not ESC" CTCF sites calculated with CTCF ChIP-seq data in ESCs (Shen et al. 2012) and NPCs (Phillips-Cremins et al. 2013). (D) Same as panel C but for 5mC density in NPCs.

Figure $3 \mathrm{~A}$ shows that the average $5 \mathrm{mC}$ density oscillated around all CTCF sites. Constitutive sites were characterized by a pronounced depletion of $5 \mathrm{mCs}$ in the interval of $\sim 100$ bp upstream of and downstream from the bound CTCF, while variable and weak CTCF sites displayed a $5 \mathrm{mC}$ enrichment peak in this region. Similar oscillations were observed for the average $5 \mathrm{hmC}$ density (Fig. 3B). The nucleosome patterns around CTCF sites showed oscillations in counter-phase with $5 \mathrm{mCs}$ (Supplemental Fig. S4C,D). This oscillatory behavior was, in general, consistent with recent reports (Kelly et al. 2012; Sun et al. 2013) and with the oscillation pattern that we found in the genome-wide analysis outside of CpG islands (Fig. 1A,B).

Importantly, the $5 \mathrm{mC}$ density in ESCs determined by bisulfite sequencing (Stadler et al. 2011) was not depleted in the middle of the "ESC not MEF" CTCF sites, as one would expect if $5 \mathrm{mCs}$ inhibit CTCF binding (Fig. 3A). In a lower-resolution DNA methylation data set measured using MeDIP in ESCs (Wilson et al. 2012), the $5 \mathrm{mC}$ density averaged over windows of $\sim 300$ bp revealed a depletion around "ESC not MEF" sites, similar to the constitutive "ESC and MEF" sites (Supplemental Fig. S4A). However, this simple relation found in ESCs was lost in MEFs (Supplemental Fig. S4B). Unlike ESCs, MEFs did not reveal a dip in $5 \mathrm{mC}$ enrichment at constitutive CTCF sites with the MeDIP data set (Wilson et al. 2012). This complicated relation was further dissected by our analysis of the high-resolution $5 \mathrm{mC}$ data in NPCs (Stadler et al. 2011), combined with the recently published CTCF ChIP-seq data set in NPCs (Fig. 3C,D; Phillips-Cremins et al. 2013). For the "ESC not NPC" binding sites, DNA methylation levels had a peak in both cell types. In contrast, the "NPC not ESC" class of sites was characterized by the $5 \mathrm{mC}$ depletion in both cell types, independently of whether CTCF was bound or not. From the above analysis, we conclude that DNA methylation anti-correlates with CTCF binding in many cases, but it is not sufficient to predict differential CTCF binding.

TET1 binding and $5 \mathrm{mC} / 5 \mathrm{hmC}$ conversion outside of $\mathrm{CpG}$ clusters correlate with labile nucleosomes

To further dissect the interplay of DNA (de)methylation, nucleosome positioning, and CTCF binding, we analyzed how the nucleosome stability near CTCF binding sites was affected by TET1, an enzyme that converts $5 \mathrm{mC}$ to $5 \mathrm{hmC}$. The TET1 binding profile in ESCs was derived from ChIP-seq data (Williams et al. 2011), and $5 \mathrm{hmC}$ patterns in ESCs were obtained from TET-assisted bisulfite mapping (Yu et al. 2012). Interestingly, $\sim 10 \%$ of TET1 ChIP-seq
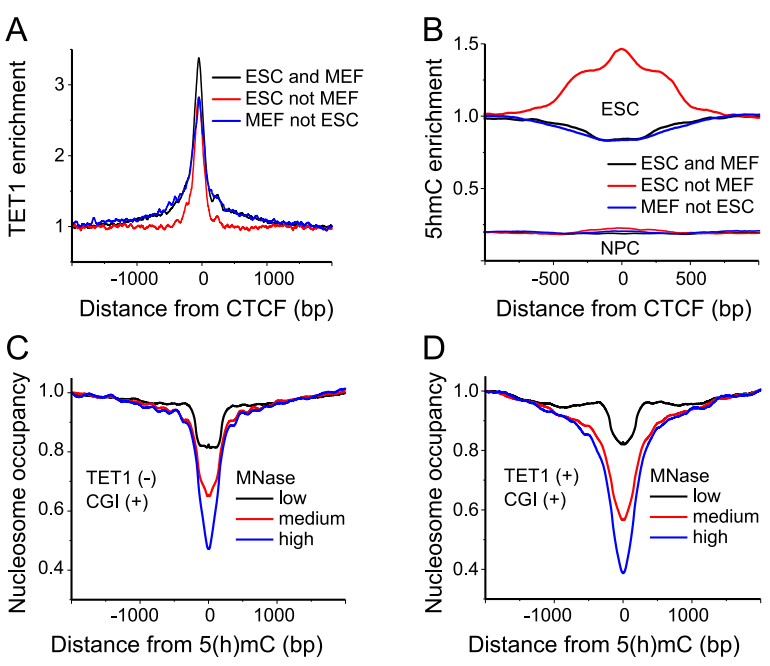

D
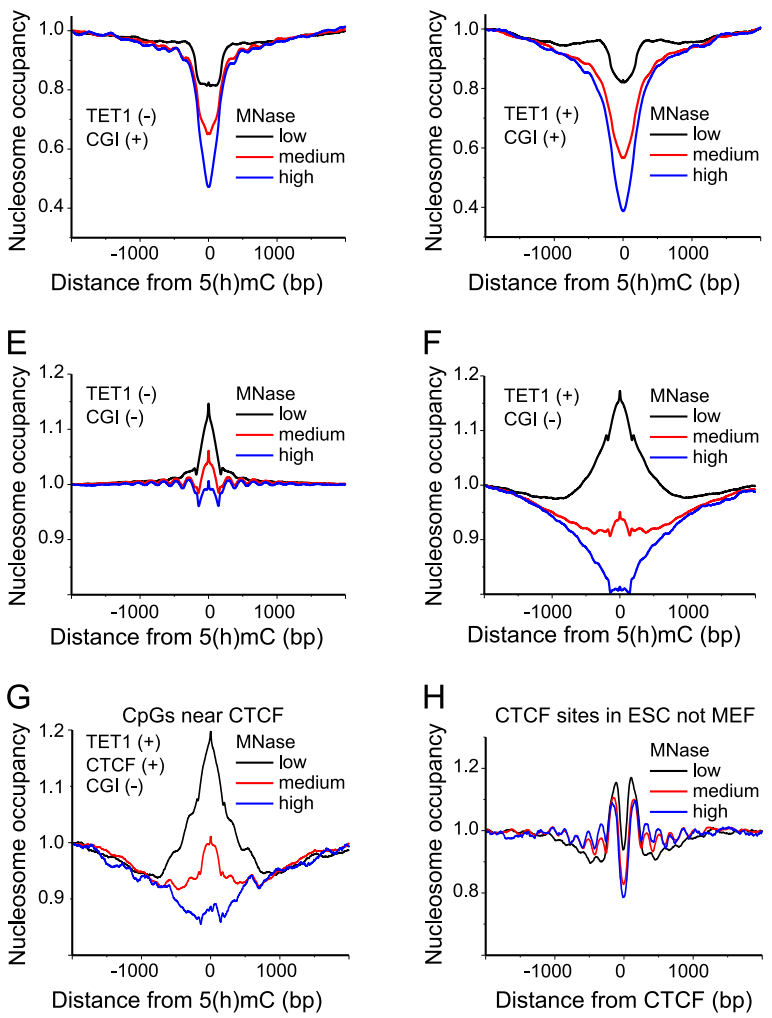

Figure 4. TET1 binding outside CGIs is linked with labile MNasesensitive nucleosomes. ( $A$ ) Average TET1 enrichment calculated from ChIP-seq data in ESCs (Williams et al. 2011) around CTCF binding sites (Shen et al. 2012). (B) Enrichment of $5 \mathrm{hmC}$ calculated from hMeDIP data in ESCs and NPCs (Tan et al. 2013) around CTCF binding sites (Shen et al. 2012; Phillips-Cremins et al. 2013). (C) Average nucleosome occupancy profiles around $5(\mathrm{~h}) \mathrm{mC}$ in the absence of TET1 within CGIs for low (black line), medium (red line), and high (blue line) MNase digestion in ESCs. Note that bisulfite sequencing does not distinguish between $5 \mathrm{mC}$ and $5 \mathrm{hmC}$. (D) Same as panel $C$ but for $5(\mathrm{~h}) \mathrm{mC}$ regions enriched with TET1. ( $E$ ) Nucleosome occupancy at 5(h)mC without TET1 outside of CGIs. Same color-coding as in panel $C$. $(F)$ Same as panel $E$ but for $5(\mathrm{~h}) \mathrm{mC}$ regions with TET1. (G) Same as in panel $F$ but only for (hydroxy)methylated CpGs that were within a 500 bp distance of bound CTCF. $(H)$ Nucleosome occupancy around CTCF sites and occupied by CTCF in ESCs but not in MEFs. Same color-coding as in panel C.

\section{Genome Research www.genome.org}


peaks overlapped with CTCF binding, resulting in TET1 enrichment for all classes of CTCF binding sites (Fig. 4A). While the level of DNA methylation changed only moderately at CTCF sites during stem cell development, the level of hydroxymethylation is dramatically different between ESCs and NPCs in general and around CTCF binding sites in particular (Fig. 4B). It is noted that the DNA methylation data used in Figure $4 \mathrm{C}-\mathrm{G}$ were determined by bisulfite sequencing (Stadler et al. 2011) and thus do not distinguish between cytosine methylation or hydroxmethylation. While in general the amount of $5 \mathrm{hmC}$ is only $5 \%-10 \%$ of the $5 \mathrm{mC}$ level in mouse embryonic stem cells, it is enriched at sites where TET1 is bound (Pastor et al. 2013). This is indicated here by using the designation $5(\mathrm{~h}) \mathrm{mC}$ in the context of Figure 4 . To evaluate the effect of TET1 on nucleosome stability, we performed an analysis of our three separate chromatin preparations for ESCs with different levels of MNase, each comprising $\sim 150$ million paired-end mononucleosomal sequencing reads. Stable nucleosomes appear as being rather independent of MNase digestion levels, while unstable nucleosomes are lost at pronounced MNase treatment. For 5(h)mCs inside CGIs, the nucleosome occupancy profiles obtained with different levels of MNase digestion converged to almost the same pattern with the characteristic nucleosome depletion in the middle (Fig. 4C,D). In contrast, nucleosome occupancy profiles around 5(h)mCs outside of CGIs showed a strong dependence on the degree of MNase digestion at TET1 sites (Fig. 4E,F). This pattern was also observed for unmethylated CpGs inside CGIs (Supplemental Fig. S7). In the presence of TET1, the nucleosome pattern of these CpGs outside of CGIs was represented by MNase-sensitive hotspots. Labile nucleosomes seen at low MNase levels were lost at higher MNase digestion (Fig. 4F). The same pattern was observed at (hydroxy)methylated CpGs near CTCF binding sites associated with TET1 (Fig. 4G). Importantly, only $6 \%$ of variable ("ESC not MEF") CTCF sites overlapped with CpG clusters, whereas this value was $21 \%$ for constitutive ("ESC and MEF") and $27 \%$ for weak ("MEF not ESC") CTCF sites. Thus, TET1-associated labile nucleosomes were enriched at variable CTCF sites (Fig. 4H), suggesting that this is linked to differential CTCF binding.

The conclusion that TET1-bound CpGs outside CGIs are characterized by labile nucleosomes was further corroborated by the corresponding heat maps of nucleosome occupancy around bound TET1 (Fig. 5A,B): Nucleosome maps obtained at low levels of MNase digestion were enriched with nucleosomes containing TET1 binding sites, while for high MNase digestion, nucleosomes became depleted at the same sites. Labile nucleosomes could be linked to the enrichment of H2A.Z (encoded by H2afz) (Supplemental Fig. S3A) and the active chromatin mark H3K4me3 (Supplemental Fig. S3B). In contrast, the inactive heterochromatin mark H3K9me3 was enriched at highly methylated CpGs and depleted at unmethylated/ low-methylated CpGs (Supplemental Fig. S3C).

To further dissect the fate of nucleosomes at the sites undergoing $5 \mathrm{mC} / 5 \mathrm{hmC}$ conversion, $5 \mathrm{hmC}$ sites were stratified according to their hydroxymethylation level of $>90 \%,>50 \%$, and $>25 \%$ obtained from the TET-assisted bisulfite mapping data (Yu et al. 2012). When increasing $5 \mathrm{hmC}$ levels, the nucleosome occupancy at the $5 \mathrm{hmC}$ site changed from a slight enrichment to a strong depletion (Fig. 5C). This indicates that the process of $5 \mathrm{mC} /$ $5 \mathrm{hmC}$ conversion is coupled to nucleosome eviction. Upon cell differentiation, the high ESC $5 \mathrm{hmC}$ levels decrease (Tan et al. 2013). This is accompanied by an increase of nucleosome occupancy, as shown in Figure 5D for sites with $>50 \% 5 \mathrm{hmC}$ in ESCs. MEFs have the lowest $5 \mathrm{hmC}$ content and the strongest nucleosome peak at those sites that were covered by labile nucleosomes when hydroxymethylated in ESCs. Conversion of $5 \mathrm{hmC}$ to $5 \mathrm{fC}$ did not show this trend (Supplemental Fig. S8), but one should be cautious due to a lower resolution of the $5 \mathrm{fC}$ data set obtained using enzymatic rather than chemical mapping (Raiber et al. 2012). Thus, nucleosomes outside of CpG clusters can be destabilized by the TET1-mediated $5 \mathrm{mC}$ to $5 \mathrm{hmC}$ conversion in ESCs or stabilized as $5 \mathrm{hmC}$ is being removed during cell differentiation to regulate accessibility for CTCF at these sites.

\section{Changes of CTCF binding during embryonic stem cell differentiation can be predicted from a quantitative model that accounts for competition with nucleosomes}

To describe the relation between variable CTCF binding and TET1dependent nucleosome rearrangements quantitatively, we developed a biophysical model for predicting genome-wide CTCF occupancy in ESCs and MEFs. To this end, we compared the average experimental CTCF occupancy profiles calculated from the raw ChIP-seq data (Fig. 6A; Shen et al. 2012) to the theoretically predicted CTCF binding profiles calculated by our TFnuc software (Methods). CTCF binding profiles calculated from its DNA sequence affinities without taking into account nucleosomes were similar to those experimentally found in ESCs (Supplemental Fig. S5A), consistent with the sensitivity/specificity analysis shown in Figure 2D. However, in MEFs, variable CTCF sites showed a loss of CTCF and a concomitant nucleosome occupancy increase, as represented for an exemplary genomic region in Supplemental Figure S5B. Without taking into account nucleosomes, the TFnuc algorithm predicted CTCF binding sites well in ESCs (AUC $=0.89$ ) but not in MEFs $($ AUC $=0.77)$ (Supplemental Fig. S5C). We then calculated the predicted CTCF occupancy profiles for each of the three classes of CTCF binding sites ("ESC and MEF," "ESC not MEF," "MEF not ESC"), taking into account competition with binding of the histone octamer. This allowed us to reproduce the experimental occupancy profiles (Fig. 6B). CTCF occupancy of constitutive and weak sites remained roughly the same in both cell types. However, for variable sites, CTCF occupancy decreased from $80 \%$ to $40 \%$, in excellent agreement with the experimentally found decrease in Figure 6A. Thus, the change of CTCF occupancy in the variable CTCF sites can be rationalized by taking into account CTCF competition with binding of the histone octamer. Note that a simple decrease of CTCF expression in differentiated cells of $\sim 4$-fold in MEFs vs. ESC (Teif et al. 2012) would not reproduce the finding that only variable "ESC not MEF" but not weak "MEF not ESC" sites became depleted of nucleosomes upon cell differentiation.

The DNA sequence motifs of constitutive and variable "ESC not MEF" sites were very similar (Fig. 6C), but the CpG content in an $\sim 1$-kb region around these sites was different (Fig. 6D). The CpG content around variable "ESC not MEF" CTCF sites was the smallest in comparison with the "ESC and MEF" and "MEF not ESC" sites (Fig. 6D). The loss of CpG enrichment in the region from 100 to $500 \mathrm{bp}$ from the center of variable CTCF sites in comparison with constitutive ones $\left(P<10^{-280}\right)$ indicates that these sites were mostly not inside $\mathrm{CpG}$ islands. Indeed, variable CTCF sites were predominantly found in FMR regions (87\%). For comparison, constitutive CTCF sites were frequently found at cisregulatory regions, with $14 \%$ in LMRs, $27 \%$ in UMRs, and only $59 \%$ in FMRs. This explains why constitutive CTCF sites at promoter/enhancer regions remain mainly unchanged during cell development, while variable CTCF sites located in the distal genomic regions outside of CGIs are differentially bound due to chromatin reorganization. 
A
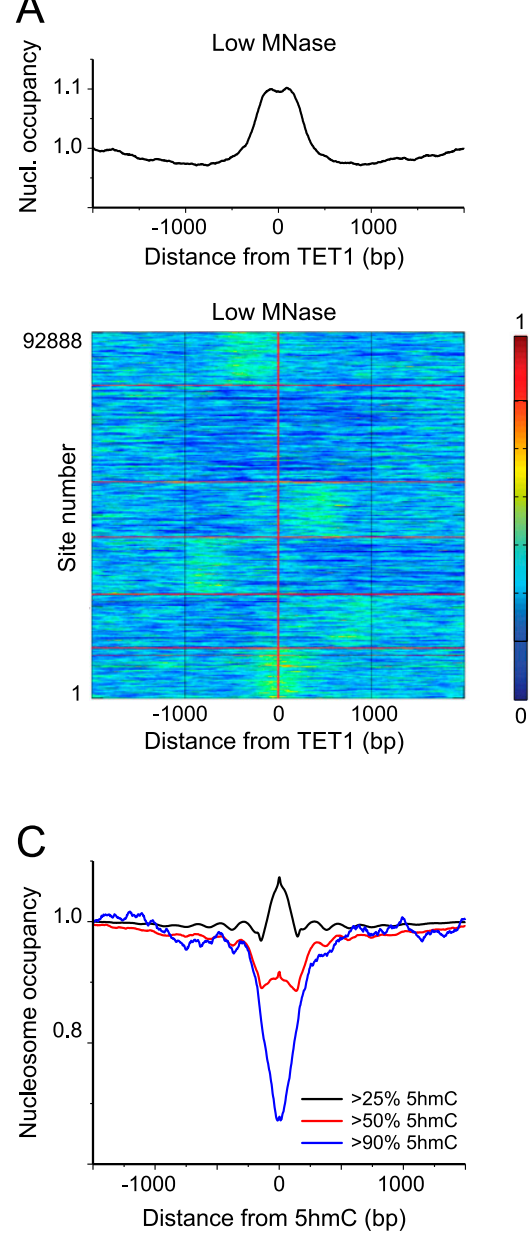

B
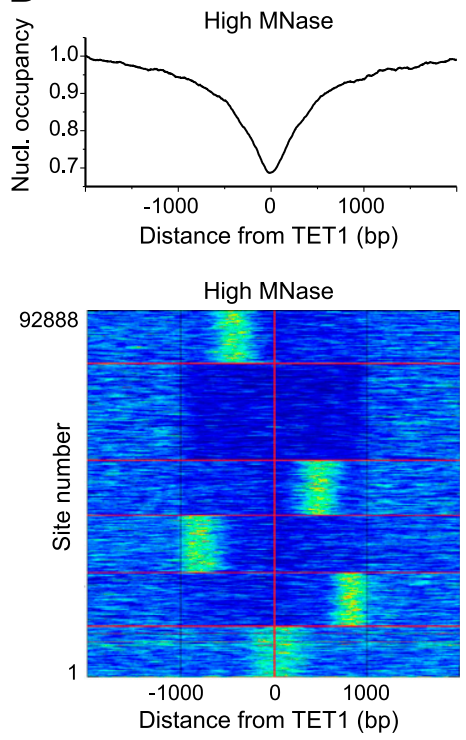

D

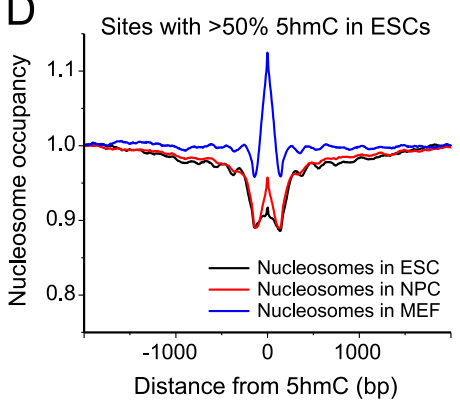

Figure 5. Nucleosome occupancy around TET1 binding sites and in relation to 5hmC levels. $(A)$ Average nucleosome occupancy (top panel) and k-means cluster plots showing nucleosome occupancy around each of 92,888 TET1 ChIP-seq peaks in ESCs (Yu et al. 2012) at low MNase digestion (bottom panel). (B) Same as panel $A$ but for high MNase digestion. (C) Aggregate plot of nucleosome occupancy around $5 \mathrm{hmC}$ sites in ESCs (Yu et al. 2012) grouped according to their $5 \mathrm{hmC}$ levels as $>25 \% 5 \mathrm{hmC}$ (black line), $>50 \% 5 \mathrm{hmC}$ (red line), and $>90 \% 5 \mathrm{hmC}$ (blue line). Upon increasing the $5 \mathrm{hmC}$ level, the nucleosome density changed from slight enrichment to nucleosome depletion, which corresponds to the nucleosome removal at a subset of these sites. $(D)$ Changes of the nucleosome occupancy during cell development (ESCs, NPCs, and MEFs) around hydroxymethylated sites in ESCs (>50\% $5 \mathrm{hmC}$ ).

The low stability of nucleosomes at variable CTCF sites appears to also involve active nucleosome translocation and other changes of the underlying chromatin states that contribute to nucleosome positioning as inferred from the following findings: (1) Bound CTCFs colocalized with chromatin remodelers such as CHD4 (also known as Mi-2beta), which as part of the NURD complex, can translocate nucleosomes and also recognize $5 \mathrm{mC}$ via associated MBD2 and MBD3 subunits (Supplemental Fig. S6B). Interestingly, variable "ESC not MEF" CTCF sites were characterized by the largest enrichment of CHD4 in ESCs, suggesting that this subset of sites undergoes the largest nucleosome turnover; (2) nucleosomes positioned at a variable CTCF site in MEFs preferably carried an H3K4me3 modification (Supplemental Fig. S4F); and (3) weak "MEF not ESC" CTCF sites covered by nucleosomes were DNA-methylated (Supplemental Fig. S4A,B) and enriched with the PRC2 subunit SUZ12 (Supplemental Fig. S6A; Hu et al. 2013a).

Thus, our results provide an explanation for differential CTCF binding between ESCs and MEFs for the "ESC not MEF" class of sites. At these loci, a TET1-dependent $5 \mathrm{mC} / 5 \mathrm{hmC}$ conversion, possibly in conjunction with active chromatin remodeling, creates MNase-sensitive labile nucleosomes in ESCs. These nucleosomes can be displaced by CTCF according to the quantitative competitive binding description introduced above. Upon cell differentiation, the levels of $5 \mathrm{hmC}$ and TET1 decrease, as these sites become DNA-methylated and adopt additional changes of their chromatin state. These processes result in higher nucleosome occupancy at the variable CTCF binding sites in MEFs, which is represented by a higher DNA binding affinity of the histone octamer in our calculations. In turn, the binding competition between CTCF and the histone octamer is shifted toward the histone octamer, and CTCF becomes depleted from these loci in MEFs.

\section{Discussion}

In order to dissect the interplay between DNA methylation, nucleosome positioning, and CTCF binding, we performed an integrative, genome-wide analysis. We mapped nucleosome positions by MNase digestion and paired-end sequencing for mononucleosome and dinucleosome samples extracted from mouse embryonic stem cells (ESCs) and their differentiated counterparts. These experiments were performed at different degrees of MNase digestion, which allowed us to determine MNase-sensitive chromatin remodeling hotspots in addition to the previously determined static nucleosome landscapes (Teif et al. 2012). By integrating these data, we resolved a puzzling controversy with respect to in vivo preferences of DNA methylation between or inside nucleosomes (Chodavarapu et al. 2010; Felle et al. 2011; Kelly et al. 2012; Li et al. 2012; Collings et al. 2013). According to our analysis, unmethylated DNA regions were characterized by an $\sim 30 \%$ lower average nucleosome density on the kb length scale and are typically inside CGIs (Supplemental Fig. S2). Most CGIs are unmethylated, but for the minority of methylated CGIs, the nucleosome was associated with the region(s) containing $5 \mathrm{mC}$ (Fig. 1D). The remaining part of the genome outside of $\mathrm{CpG}$ islands has a very different relative distribution of $5 \mathrm{mC}$ and nucleosomes: The average $5 \mathrm{mC}$ density oscillates in such a way that it is minimal in the nucleosome center and increases toward the ends of the nucleosome, reaching its maximum in the linker regions between nucleosomes (Fig. 1). Interestingly, the $\mathrm{CpG}$ density around nucleosome centers also undergoes regular oscillations with the period equal to the nucleosome repeat length (Supplemental Fig. S1). This type of $5 \mathrm{mC}$ oscillations revealed here in mouse has also been reported for plants (Cokus et al. 2008) and recently for several other eukaryotes (Huff and Zilberman 2014) and adds a new layer of the genomic code complexity. The oscillations of both CpGs and $5 \mathrm{mC}$ density

\section{Genome Research} www.genome.org 

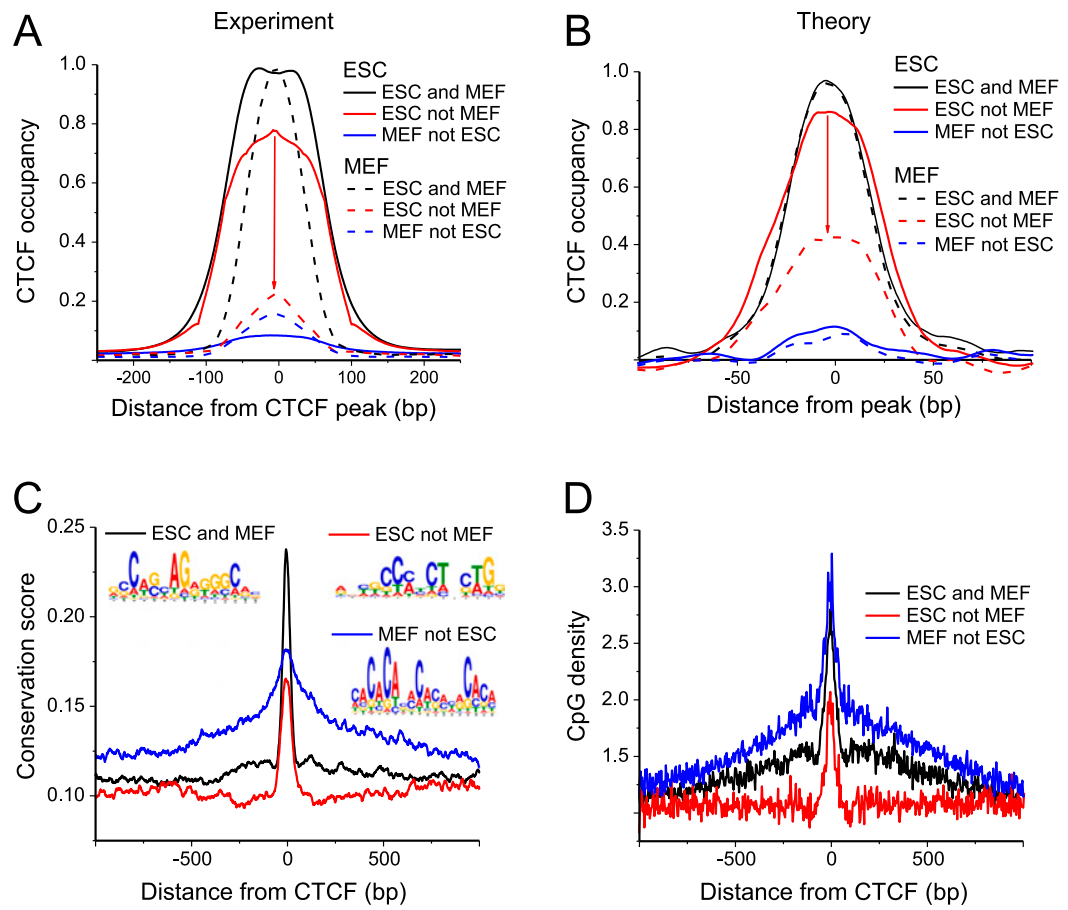

Figure 6. Quantitative model to predict CTCF occupancy changes due to competition with nucleosomes. (A) Experimentally observed occupancy of CTCF binding sites in ESCs and MEFs derived from ChIP-seq peak heights (Shen et al. 2012). Three subsets of CTCF sites can be distinguished: constitutive sites bound in both cell types (ESC and MEF), variable sites predominantly bound by CTCF in ESCS (ESC not MEF), and weak sites which are slightly more strongly bound in differentiated cells (MEF not ESC). (B) Predicted CTCF binding site occupancy in ESCs and MEFs when accounting for nucleosome positioning for the three binding site classes shown in panel $A$. (C) Conservation score of CTCF DNA binding sequence motifs for constitutive, variable, and weak binding sites. $(D)$ Average CpC density at CTCF sites. The variable sites in the "ESC not MEF" class are located preferentially outside of CGls.

were anti-correlated with nucleosome occupancy outside of CGIs. The conclusions obtained with the mononucleosome fraction were confirmed for the dinucleosome fraction extracted after moderate MNase digestion, which showed an $\sim 20 \% 5 \mathrm{mC}$ enrichment in the linker regions between nucleosomes (Fig. 1B). Inasmuch as DNA methylation in the linker region is recognized by methyl-binding proteins, it could contribute to enriching a dinucleosome particle during the MNase digestion step. Such a complex consisting of a $5 \mathrm{mC}$-recognizing protein binding to the linker DNA between two nucleosomes might be important for the formation of heterochromatic regions (Bulut-Karslioglu et al. 2012) and intron/exon recognition during the cotranscriptional splicing (Gelfman et al. 2013).

With respect to the CTCF binding site selection in a single cell type (ESC), we showed that CTCF binding preferences are primarily due to the DNA sequence and can be well predicted based on the DNA sequence alone (Fig. 2; Supplemental Fig. S5). Although DNA methylation and nucleosome positions did correlate with CTCF binding in ESCs, this was rather a downstream effect of CTCF binding, consistent with previous observations (Stadler et al. 2011; Feldmann et al. 2013). Interestingly, the DNA methylation state was previously used by Stadler et al. to improve CTCF binding prediction from $\sim 40 \%$ to $\sim 50 \%$ of the binding site variability (Stadler et al. 2011). In our calculations performed for ESCs, the incorporation of the knowledge of DNA methylation led to only a marginal improvement with respect to the pure PWM model (from $89 \%$ to $90 \%$ in Fig. 2D). Possibly, our model for CTCF binding in ESCs was initially better optimized in the absence of DNA methylation information (AUC $=0.89$ ), so that including binding sites well without taking into account nucleosomes. It failed to do so in MEFs (Supplemental Fig. S5C), where nucleosome rearrangement impeded CTCF binding to the variable sites (Fig. 6B; Supplemental Fig. S5B). This suggests that ESCs have more dynamic chromatin, where CTCF can displace nucleosomes more easily. Such a global difference in chromatin dynamics is consistent with the general notion of ESC chromatin as being more "open" in comparison with differentiated cells (GasparMaia et al. 2011; Biran and Meshorer 2012). Our study suggests a novel mechanistic explanation to rationalize this effect, in addition to the previously identified differences of CTCF binding during ESC differentiation, as depicted in Figure 7. CTCF binding inside CGIs is mostly invariant and determined by the DNA sequence (Fig. 7A). It comprises strong constitutive CTCF sites that remain unmethylated and occupied by CTCF both in ESCs and MEFs. The weak sites in these regions are mostly not bound by CTCF in both cell types. However, outside of CpG islands, binding of TET creates remodeling hotspots with labile MNase-sensitive nucleosomes in ESCs (Figs. 4, 5). These nucleosomes are prone to become evicted upon $5 \mathrm{mC}$ conversion to $5 \mathrm{hmC}$ (Fig. 5C) or become stabilized upon $5 \mathrm{hmC}$ removal during cell differentiation. Thus, TET1-dependent $5 \mathrm{mC} / 5 \mathrm{hmC}$ conversion opens up chromatin and creates labile nucleosomes that can be subsequently displaced by CTCF in ESCs, as depicted in Figure 7B. On the other hand, in differentiated cells, 5hmC and TET1 levels decrease and no longer support CTCF competition with the nucleosome at the variable sites that lose CTCF during differentiation.

Are there other factors in addition to nucleosomes that might influence the selection of CTCF binding sites during stem 
A

Inside $\mathrm{CpG}$ islands

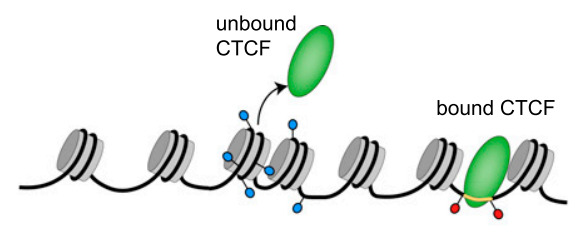

B

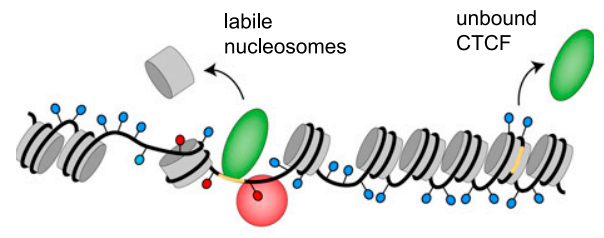

$95 \mathrm{mC}$
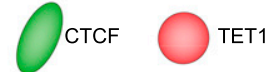

Figure 7. Model rationalizing the linkages between $5 \mathrm{mC}, 5 \mathrm{hmC}$ TET1-, and CTCF-binding with nucleosome positioning inside and outside of $\mathrm{CpG}$ islands. $(A)$ Inside $\mathrm{CpG}$ islands, most $\mathrm{CpG}$ s are unmethylated and have low nucleosome occupancy. The small fraction of $\mathrm{CpG}$ s in CGls that are methylated has a nucleosome positioned preferably within the DNA methylation sites. CTCF binding inside CGIs is mostly invariant and determined by the DNA sequence: Strong constitutive CTCF sites stay unmethylated and bound by CTCF and its cobinders during the cell development, while weak sites in these regions are mostly not bound by CTCF in both ESCs and differentiated cells. (B) Outside of CGls, the genomic DNA is mostly methylated at CpGs. In relation to nucleosome positioning, the following features were found: DNA methylation density is lowest in the middle of the nucleosome, smoothly increases toward the nucleosome entry/exit, and reaches a maximum between nucleosomes. In these regions, TET1 binding creates MNase-sensitive labile nucleosomes, which are being removed/translocated during the process of $5 \mathrm{mC}$ to $5 \mathrm{hmC}$ conversion. Variable CTCF sites are found preferentially outside CGIs, where active, TET1-dependent hydroxymethylation and associated nucleosome repositioning promotes CTCF binding. As CpGs in these regions change to a methylated state during stem cell differentiation, the formation of stably bound nucleosomes leads to a loss of CTCF at these sites.

cell development? We have tested proteins whose ChIP-seq data for ESCs are currently available in the literature, and found several potential CTCF binding partners including MECP2 (Baubec et al. 2013), MBD1 (Baubec et al. 2013), YY1 (Vella et al. 2012), and cohesin (Supplemental Fig. S6C; Kagey et al. 2010), which colocalized with CTCF. However, they all showed stronger enrichments around constitutive versus variable CTCF sites, which could not explain CTCF binding variability. For proteins whose ChIP-seq data are not available in ESCs, we applied a bioinformatics analysis suggested recently (Luu et al. 2013), and indeed found several of their $5 \mathrm{mC}$-sensitive motifs enriched around the "ESC not MEF" and "ESC and MEF" sites. Interestingly, the significance of this enrichment for GATA1, FOXO4, and TCF3, as given by $P$-values calculated using the TRAP algorithm (Roider et al. 2007), was consistently several orders of magnitude stronger for "ESC not MEF" vs. "ESC and MEF." This might suggest that additional complex mechanisms can be at play, involving many TFs whose concentrations change during the cell development. For CTCF, our data suggest that the $\mathrm{TET} 1 / 5 \mathrm{hmC} / 5 \mathrm{mC}$-dependent nucleosome repositioning remains the main mechanism that drives its dif- ferential binding site selection during stem cell development. The novel linkage between these factors and DNA binding site accessibility could be relevant also for other TFs during stem cell differentiation or ligand-activated TF binding (John et al. 2011).

\section{Methods}

\section{MNase-seq}

MNase-seq experiments for analysis of mononucleosomes and dinucleosomes were conducted as described previously (Teif et al. 2012). Briefly, embryonic stem cells from 129P2/Ola mice were cultured in ESGRO complete medium (Millipore), harvested, and resuspended in low-salt buffer $(10 \mathrm{mM}$ Hepes, $\mathrm{pH} 8,10 \mathrm{mM} \mathrm{KCl}$, $0.5 \mathrm{mM} \mathrm{DTT})$ at $4^{\circ} \mathrm{C}$. After disruption of the cells with a douncer, the nuclei were collected by centrifugation and washed once with the MNase Buffer (10 mM Tris- $\left.\mathrm{HCl}, \mathrm{pH} 7.5,10 \mathrm{mM} \mathrm{CaCl}_{2}\right)$, resuspended in the MNase Buffer, and digested with 0.5 units MNase per microliter (Fermentas) and incubation for $6-11 \mathrm{~min}$ at $37^{\circ} \mathrm{C}$. The MNase digestion was stopped by putting the samples on ice and adding EDTA to a concentration of $10 \mathrm{mM}$. After digestion with 0.1 $\mu \mathrm{gl}^{-1}$ RNase A (Fermentas) and removal of protein by phenol and chloroform extraction, the DNA was ethanol-precipitated, and the resulting DNA pellet was dissolved in $\mathrm{H}_{2} \mathrm{O}$. DNA fragments corresponding to mononucleosomes or dinucleosomes were separated on a $2 \%$ agarose gel using an E-Gel electrophoresis system (Life Technologies). The libraries for sequencing were prepared according to the standard Illumina protocol. High-throughput paired-end sequencing of 50-bp read length was performed on the Illumina HiSeq 2000 platform at the DKFZ sequencing core facility in Heidelberg, Germany. We obtained $\sim 150$ million nucleosome positions per sequencing reaction and used in the final analysis two biological replicate experiments for each cell condition.

\section{Chromatin immunoprecipitation}

ChIP-seq experiments were conducted as described previously (Teif et al. 2012). For each sample, $1 \times 10^{6}$ cells were cross-linked with $1 \%$ PFA, and cell nuclei were prepared using a swelling buffer $(25 \mathrm{mM}$ Hepes, pH 7.8, $1 \mathrm{mM} \mathrm{MgCl}_{2}, 10 \mathrm{mM} \mathrm{KCl}, 0.1 \%$ NP-40, 1 mM DTT). Chromatin was sheared to mononucleosomal fragments. After IgG preclearance, the sheared chromatin was incubated with $4 \mu \mathrm{g}$ of either H3K9me3 (Abcam ab8898) or H3K4me3 (Abcam ab8580) antibody overnight. After washes with sonication $(10 \mathrm{mM}$ Tris- $\mathrm{HCl}$, pH 8.0, $200 \mathrm{mM} \mathrm{NaCl}, 1 \mathrm{mM}$ EDTA, 0.5\% N-lauroylsarcosine, $0.1 \%$ Na-deoxycholate), high-salt-buffer (50 mM Hepes, pH 7.9, $500 \mathrm{mM}$ $\mathrm{NaCl}, 1 \mathrm{mM}$ EDTA, 1\% Triton X-100, 0.1\% Na-deoxycholate, $0.1 \%$ SDS), lithium buffer (20 mM Tris-HCl, pH 8.0, 1 mM EDTA, $250 \mathrm{mM}$ $\mathrm{LiCl}, 0.5 \% \mathrm{NP}-40,0.5 \%$ Na-deoxycholate) and $10 \mathrm{mM}$ Tris- $\mathrm{HCl}$, chromatin was eluted from the protein $\mathrm{G}$ magnetic beads and the crosslink was reversed overnight. After RNase A and proteinase $\mathrm{K}$ digestion, DNA was purified and cloned in a barcoded sequencing library for the Illumina HiSeq 2000 sequencing platform (single reads of 50-bp length).

\section{CTCF binding site classification}

The initial distinction between the classes of CTCF binding sites in ESCs and MEFs was done based on the peak calling performed previously (Shen et al. 2012), and the following intersection of the areas of the peaks extended to 1000 bp using the "intersectBED" function of the BEDTools program (Quinlan and Hall 2010). As a result, three classes of sites were identified: "ESC and MEF,"

\section{Genome Research www.genome.org}


"ESC not MEF," and "MEF not ESC." CTCF occupancy was normalized to have the average value for "ESC and MEF" sites equal to unity, which was denoted as $100 \%$ occupancy. Filtering of the "ESC and MEF" and "MEF not ESC" sites was performed to exclude sites which have $>10 \%$ of CTCF occupancy but were not found as peaks in the initial ENCODE peak calling. The resulting "MEF not ESC" class appeared to represent sites which are weak both in MEFs and ESCs. In the case of NPCs, we did peak calling of the raw ChIP-seq data from Phillips-Cremins et al. (2013) using MACS (Zhang et al. 2008 ) and then applied the same procedure as above.

\section{Calculation of experimental aggregate profiles}

All data sets used in this study were preprocessed with Perl scripts as follows: For paired-end MNase-seq, coordinates of each read start and end were used, while for ChIP-seq analysis, reads were extended by the average DNA fragment length. In the case of bisulfite sequencing, individual CpG coordinates from a previously published data set (Stadler et al. 2011) were used for the calculation of two types of coverage files corresponding to unmethylated CpGs (methylation $<10 \%$ ), and methylated CpGs (methylation $>50 \%$ ). At the next step, aggregate plots were calculated by summing up all occupancy maps of the individual regions around a given feature. For example, in the case of the calculation of nucleosome occupancy around CpGs (Fig. 1), all annotated mouse CpGs were used for the analysis. When $5 \mathrm{mC}$ and CpG patterns were calculated around nucleosome dyads, the midpoints of all paired-end mono- or dinucleosome reads were used in the analysis. To facilitate calculations shown in Figure 1, A and $\mathrm{B}$, we used only reads from chromosome 19 . Including regions from other chromosomes did not change the results. Due to the large number of genomic regions averaged in the aggregate plots, the differences between all plotted curves are statistically significant. For example, in Figure 4A, the TET1 peak around "ESC not MEF" sites is more narrow than the peak around "ESC and MEF" sites, with $P=0.0097$ [two-tailed $t$-test calculated for the ChIP-seq signal averaged with a 10 -bp step in the window $(-800$; $-300)$ from the peak center].

\section{Calculation of protein-DNA binding from DNA sequence}

CTCF binding maps were calculated with the software TFnuc that implements the approach described in our previous works (Teif and Rippe 2012; Teif et al. 2013). The program takes as input the DNA sequence, concentrations, and sequence-specific binding constants for transcription factors, as well as cell type-specific nucleosome coverage, and computes binding maps (binding probability distributions) for a given set of proteins to a defined DNA region as introduced elsewhere (Teif 2007, 2010). Average CTCF binding profiles were calculated by summing up binding maps for all individual genomic regions (FMR, LMR, UMR regions in the case of Figure 3C, and regions around CTCF binding sites in the case of Figure 6, B and D). The estimation of sequence-specific binding affinities $K(\mathrm{CTCF})$ is based on the known proportionality between PWM scores and the binding probability of a TF to DNA (Roider et al. 2007). The PWM for CTCF was previously determined from ChIP-seq data by Chen et al. (2008), and the latter data set did not overlap with the data set from the Mouse ENCODE Project used in our study (Shen et al. 2012). The parameterization of our model was implemented in analogy to the TRAP algorithm (Roider et al. 2007), using 300 random genomic regions and requiring the best Pearson's pairwise correlation coefficient of predicted CTCF binding probabilities with the experimental occupancies from the Mouse ENCODE Project data (Shen et al. 2012). The following optimal parameters were used as input in the TFnuc program: binding constant $K(\mathrm{CTCF})=10^{9} \mathrm{M}^{-1}$, concentration $[\mathrm{CTCF}]=10^{-6} \mathrm{M}$, the energy mismatch parameter $\lambda=1.5$. To facilitate comparison with previous results, the effect of nucleosome occupancy on CTCF binding was introduced as described previously (Goh et al. 2010) in the form $K(\mathrm{CTCF})_{\text {modif }}=K(C T C F) \times$ $1.5^{- \text {nuc_occup }}$, where nuc_occup is the nucleosome occupancy at a given position taken from MNase-seq experiments.

\section{Published data sets used in the calculations}

The following published data sets have been used in the calculations: GSE40951 (mononucleosomes in ESCs and MEFs, MNaseseq); GSE30206 (5mC in ESCs and NPCs, Bis-seq); GSM882244 (5hmC in ESCs, Tet-assisted Bis-seq); GSE27468 (5mC in ESCs and MEFs, MeDIP); GSE39237 (H2A.Z in ESCs, ChIP-seq); GSM687289 (Mi-2 $\beta$ in ESCs, ChIP-seq); GSM1002268 (5hmC in ESCs and NPCs, hMeDIP); GSE24843 (TET1 in ESCs, ChIP-seq); GSE36203 (CTCF in NPCs, ChIP-seq); GSM918743 (CTCF in ESCs, ChIP-seq); GSM918748 (CTCF in MEFs, ChIP-seq); GSM970527 (SUZ12 in ESCs); GSE22557 (cohesin in ESCs). A detailed list of the data sources is provided in Supplemental Table S1F.

\section{Data access}

MNase-seq and ChIP-seq data produced in the current study have been submitted to the NCBI Gene Expression Omnibus (GEO; http:// www.ncbi.nlm.nih.gov/geo/) under accession number GSE56938.

\section{Acknowledgments}

We thank Dirk Schübeler for stimulating discussions and sharing unpublished data, and Dario Beraldi, Yin Shen, Michael Stadler, and Gareth Wilson for help and explanations about their published data sets. Part of this work was funded within the project EpiGenSys by the German Federal Ministry of Education and Research (BMBF) as a partner of the ERASysBio+ initiative in the EU FP7 ERA-NET Plus program (0315712A). Computational resources and data storage were provided via grants from the BMBF (01IG07015G, Services@MediGRID) and the German Research Foundation (DFG INST 295/27-1). V.B.T. acknowledges the support from the Heidelberg Center for Modeling and Simulation in the Biosciences (BIOMS) and a DKFZ Intramural Grant.

\section{References}

Baubec T, Ivanek R, Lienert F, Schubeler D. 2013. Methylation-dependent and -independent genomic targeting principles of the MBD protein family. Cell 153: 480-492.

Biran A, Meshorer E. 2012. Concise review: chromatin and genome organization in reprogramming. Stem Cells 30: 1793-1799.

Bulut-Karslioglu A, Perrera V, Scaranaro M, de la Rosa-Velazquez IA, van de Nobelen S, Shukeir N, Popow J, Gerle B, Opravil S, Pagani M, et al. 2012. A transcription factor-based mechanism for mouse heterochromatin formation. Nat Struct Mol Biol 19: 1023-1030.

Chen X, Xu H, Yuan P, Fang F, Huss M, Vega VB, Wong E, Orlov YL, Zhang W, Jiang J, et al. 2008. Integration of external signaling pathways with the core transcriptional network in embryonic stem cells. Cell 133: 1106-1117.

Chodavarapu RK, Feng S, Bernatavichute YV, Chen PY, Stroud H, Yu Y, Hetzel JA, Kuo F, Kim J, Cokus SJ, et al. 2010. Relationship between nucleosome positioning and DNA methylation. Nature 466: 388-392.

Choy MK, Movassagh M, Goh HG, Bennett MR, Down TA, Foo RS. 2010. Genome-wide conserved consensus transcription factor binding motifs are hyper-methylated. BMC Genomics 11: 519.

Cokus SJ, Feng S, Zhang X, Chen Z, Merriman B, Haudenschild CD, Pradhan S, Nelson SF, Pellegrini M, Jacobsen SE. 2008. Shotgun bisulphite sequencing of the Arabidopsis genome reveals DNA methylation patterning. Nature 452: 215-219. 
Collings CK, Waddell PJ, Anderson JN. 2013. Effects of DNA methylation on nucleosome stability. Nucleic Acids Res 41: 2918-2931.

Feldmann A, Ivanek R, Murr R, Gaidatzis D, Burger L, Schubeler D. 2013 Transcription factor occupancy can mediate active turnover of DNA methylation at regulatory regions. PLoS Genet 9: e1003994.

Felle M, Hoffmeister H, Rothammer J, Fuchs A, Exler JH, Langst G. 2011. Nucleosomes protect DNA from DNA methylation in vivo and in vitro. Nucleic Acids Res 39: 6956-6969.

Gaspar-Maia A, Alajem A, Meshorer E, Ramalho-Santos M. 2011. Open chromatin in pluripotency and reprogramming. Nat Rev Mol Cell Biol 12: 36-47.

Gelfman S, Cohen N, Yearim A, Ast G. 2013. DNA-methylation effect on cotranscriptional splicing is dependent on GC architecture of the exonintron structure. Genome Res 23: 789-799.

Goh WS, Orlov Y, Li J, Clarke ND. 2010. Blurring of high-resolution data shows that the effect of intrinsic nucleosome occupancy on transcription factor binding is mostly regional, not local. PLoS Comput Biol 6: e1000649.

Gordan R, Hartemink AJ, Bulyk ML. 2009. Distinguishing direct versus indirect transcription factor-DNA interactions. Genome Res 19: 2090-2100.

Hackenberg M, Previti C, Luque-Escamilla PL, Carpena P, Martinez-Aroza J, Oliver JL. 2006. CpGcluster: a distance-based algorithm for CpG-island detection. BMC Bioinformatics 7: 446

Handoko L, Xu H, Li G, Ngan CY, Chew E, Schnapp M, Lee CW, Ye C, Ping JL, Mulawadi F, et al. 2011. CTCF-mediated functional chromatin interactome in pluripotent cells. Nat Genet 43: 630-638.

He X, Chatterjee R, John S, Bravo H, Sathyanarayana BK, Biddie SC, Fitzgerald PC, Stamatoyannopoulos JA, Hager GL, Vinson C. 2013. Contribution of nucleosome binding preferences and co-occurring DNA sequences to transcription factor binding. BMC Genomics 14: 428.

Hu G, Cui K, Northrup D, Liu C, Wang C, Tang Q, Ge K, Levens D, CraneRobinson C, Zhao K. 2013a. H2A.Z facilitates access of active and repressive complexes to chromatin in embryonic stem cell self-renewal and differentiation. Cell Stem Cell 12: 180-192.

Hu S, Wan J, Su Y, Song Q, Zeng Y, Nguyen HN, Shin J, Cox E, Rho HS, Woodard C, et al. 2013b. DNA methylation presents distinct binding sites for human transcription factors. eLife 2: e00726.

Huff JT, Zilberman D. 2014. Dnmt1-independent CG methylation contributes to nucleosome positioning in diverse eukaryotes. Cell 156: 1286-1297.

John S, Sabo PJ, Thurman RE, Sung MH, Biddie SC, Johnson TA, Hager GL, Stamatoyannopoulos JA. 2011. Chromatin accessibility pre-determines glucocorticoid receptor binding patterns. Nat Genet 43: 264-268.

Kagey MH, Newman JJ, Bilodeau S, Zhan Y, Orlando DA, van Berkum NL, Ebmeier CC, Goossens J, Rahl PB, Levine SS, et al. 2010. Mediator and cohesin connect gene expression and chromatin architecture. Nature 467: 430-435.

Kaplan T, Li XY, Sabo PJ, Thomas S, Stamatoyannopoulos JA, Biggin MD, Eisen MB. 2011. Quantitative models of the mechanisms that control genome-wide patterns of transcription factor binding during early Drosophila development. PLoS Genet 7: e1001290.

Kasowski M, Kyriazopoulou-Panagiotopoulou S, Grubert F, Zaugg JB, Kundaje A, Liu Y, Boyle AP, Zhang QC, Zakharia F, Spacek DV, et al. 2013. Extensive variation in chromatin states across humans. Science 342: $750-752$.

Kelly TK, Liu Y, Lay FD, Liang G, Berman BP, Jones PA. 2012. Genome-wide mapping of nucleosome positioning and DNA methylation within individual DNA molecules. Genome Res 22: 2497-2506.

Li XY, Thomas S, Sabo PJ, Eisen MB, Stamatoyannopoulos JA, Biggin MD. 2011. The role of chromatin accessibility in directing the widespread, overlapping patterns of Drosophila transcription factor binding. Genome Biol 12: R34.

Li Z, Gadue P, Chen K, Jiao Y, Tuteja G, Schug J, Li W, Kaestner KH. 2012. Foxa2 and H2A.Z mediate nucleosome depletion during embryonic stem cell differentiation. Cell 151: $1608-1616$.

Lickwar CR, Mueller F, Hanlon SE, McNally JG, Lieb JD. 2012. Genome-wide protein-DNA binding dynamics suggest a molecular clutch for transcription factor function. Nature 484: 251-255.

Locke G, Tolkunov D, Moqtaderi Z, Struhl K, Morozov AV. 2010. Highthroughput sequencing reveals a simple model of nucleosome energetics. Proc Natl Acad Sci 107: 20998-21003.

Luu PL, Scholer HR, Arauzo-Bravo MJ. 2013. Disclosing the crosstalk among DNA methylation, transcription factors, and histone marks in human pluripotent cells through discovery of DNA methylation motifs. Genome Res 23: 2013-2029.

McVicker G, van de Geijn B, Degner JF, Cain CE, Banovich NE, Raj A, Lewellen N, Myrthil M, Gilad Y, Pritchard JK. 2013. Identification of genetic variants that affect histone modifications in human cells. Science 342: 747-749.

Merkenschlager M, Odom DT. 2013. CTCF and cohesin: linking gene regulatory elements with their targets. Cell 152: 1285-1297.
Narlikar L, Gordan R, Hartemink AJ. 2007. A nucleosome-guided map of transcription factor binding sites in yeast. PLoS Comput Biol 3: e215.

Pastor WA, Aravind L, Rao A. 2013. TETonic shift: biological roles of TET proteins in DNA demethylation and transcription. Nat Rev Mol Cell Biol 14: $341-356$.

Phillips-Cremins JE, Sauria ME, Sanyal A, Gerasimova TI, Lajoie BR, Bell JS, Ong CT, Hookway TA, Guo C, Sun Y, et al. 2013. Architectural protein subclasses shape 3D organization of genomes during lineage commitment. Cell 153: 1281-1295.

Plasschaert RN, Vigneau S, Tempera I, Gupta R, Maksimoska J, Everett L, Davuluri R, Mamorstein R, Lieberman PM, Schultz D, et al. 2013. CTCF binding site sequence differences are associated with unique regulatory and functional trends during embryonic stem cell differentiation. Nucleic Acids Res 42: 774-789.

Portela A, Liz J, Nogales V, Setien F, Villanueva A, Esteller M. 2013. DNA methylation determines nucleosome occupancy in the 5 '-CpG islands of tumor suppressor genes. Oncogene 32: 5421-5428.

Quinlan AR, Hall IM. 2010. BEDTools: a flexible suite of utilities for comparing genomic features. Bioinformatics 26: $841-842$.

Raiber EA, Beraldi D, Ficz G, Burgess HE, Branco MR, Murat P, Oxley D, Booth MJ, Reik W, Balasubramanian S. 2012. Genome-wide distribution of 5formylcytosine in embryonic stem cells is associated with transcription and depends on thymine DNA glycosylase. Genome Biol 13: R69.

Raveh-Sadka T, Levo M, Segal E. 2009. Incorporating nucleosomes into thermodynamic models of transcription regulation. Genome Res 19: 1480-1496.

Razin A, Cedar H. 1977. Distribution of 5-methylcytosine in chromatin. Proc Natl Acad Sci 74: 2725-2728.

Reddington JP, Pennings S, Meehan RR. 2013. Non-canonical functions of the DNA methylome in gene regulation. Biochem J 451: 13-23.

Roider HG, Kanhere A, Manke T, Vingron M. 2007. Predicting transcription factor affinities to DNA from a biophysical model. Bioinformatics 23: 134-141.

Shen Y, Yue F, McCleary DF, Ye Z, Edsall L, Kuan S, Wagner U, Dixon J, Lee L, Lobanenkov VV, et al. 2012. A map of the cis-regulatory sequences in the mouse genome. Nature 488: 116-120.

Shen L, Wu H, Diep D, Yamaguchi S, D'Alessio AC, Fung HL, Zhang K, Zhang Y. 2013. Genome-wide analysis reveals TET- and TDG-dependent 5-methylcytosine oxidation dynamics. Cell 153: 692-706.

Smith ZD, Meissner A. 2013. DNA methylation: roles in mammalian development. Nat Rev Genet 14: 204-220.

Song CX, Szulwach KE, Dai Q, Fu Y, Mao SQ, Lin L, Street C, Li Y, Poidevin M, $\mathrm{Wu} \mathrm{H}$, et al. 2013. Genome-wide profiling of 5-formylcytosine reveals its roles in epigenetic priming. Cell 153: 678-691.

Spruijt CG, Gnerlich F, Smits AH, Pfaffeneder T, Jansen PW, Bauer C, Munzel M, Wagner M, Muller M, Khan F, et al. 2013. Dynamic readers for 5-(hydroxy)methylcytosine and its oxidized derivatives. Cell 152: 11461159 .

Stadler MB, Murr R, Burger L, Ivanek R, Lienert F, Scholer A, van Nimwegen E, Wirbelauer C, Oakeley EJ, Gaidatzis D, et al. 2011. DNA-binding factors shape the mouse methylome at distal regulatory regions. Nature 480: $490-495$.

Sun Z, Terragni J, Borgaro JG, Liu Y, Yu L, Guan S, Wang H, Sun D, Cheng X, Zhu Z, et al. 2013. High-resolution enzymatic mapping of genomic 5-hydroxymethylcytosine in mouse embryonic stem cells. Cell Reports 3: $567-576$.

Tan L, Xiong L, Xu W, Wu F, Huang N, Xu Y, Kong L, Zheng L, Schwartz L, Shi Y, et al. 2013. Genome-wide comparison of DNA hydroxymethylation in mouse embryonic stem cells and neural progenitor cells by a new comparative hMeDIP-seq method. Nucleic Acids Res 41: e84.

Teif VB. 2007. General transfer matrix formalism to calculate DNA-proteindrug binding in gene regulation: application to $\mathrm{O}_{R}$ operator of phage $\lambda$. Nucleic Acids Res 35: e80.

Teif VB. 2010. Predicting gene-regulation functions: lessons from temperate bacteriophages. Biophys J 98: 1247-1256.

Teif VB, Rippe K. 2012. Calculating transcription factor binding maps for chromatin. Brief Bioinform 13: 187-201.

Teif VB, Vainstein E, Marth K, Mallm J-P, Caudron-Herger M, Höfer T, Rippe K. 2012. Genome-wide nucleosome positioning during embryonic stem cell development. Nat Struct Mol Biol 19: 1185-1192.

Teif VB, Erdel F, Beshnova DA, Vainshtein Y, Mallm JP, Rippe K. 2013. Taking into account nucleosomes for predicting gene expression. Methods 62: 26-38.

Thomson JP, Skene PJ, Selfridge J, Clouaire T, Guy J, Webb S, Kerr AR, Deaton A, Andrews R, James KD, et al. 2010. CpG islands influence chromatin structure via the CpG-binding protein Cfp1. Nature 464: 1082-1086.

Van Bortle K, Corces VG. 2013. The role of chromatin insulators in nuclear architecture and genome function. Curr Opin Genet Dev 23: 212-218.

Vella P, Barozzi I, Cuomo A, Bonaldi T, Pasini D. 2012. Yin Yang 1 extends the $M y c$-related transcription factors network in embryonic stem cells. Nucleic Acids Res 40: 3403-3418.

\section{Genome Research}

www.genome.org 
Nucleosome positioning, DNA methylation, and CTCF

Wang H, Maurano MT, Qu H, Varley KE, Gertz J, Pauli F, Lee K, Canfield T, Weaver M, Sandstrom R, et al. 2012. Widespread plasticity in CTCF occupancy linked to DNA methylation. Genome Res 22: 1680-1688.

Wasson T, Hartemink AJ. 2009. An ensemble model of competitive multifactor binding of the genome. Genome Res 19: 2101-2112.

Wiench M, John S, Baek S, Johnson TA, Sung MH, Escobar T, Simmons CA, Pearce KH, Biddie SC, Sabo PJ, et al. 2011. DNA methylation status predicts cell type-specific enhancer activity. EMBO J 30: 30283039.

Williams K, Christensen J, Pedersen MT, Johansen JV, Cloos PA, Rappsilber J, Helin K. 2011. TET1 and hydroxymethylcytosine in transcription and DNA methylation fidelity. Nature 473: $343-348$.

Wilson GA, Dhami P, Feber A, Cortazar D, Suzuki Y, Schulz R, Schar P, Beck S. 2012. Resources for methylome analysis suitable for gene knockout studies of potential epigenome modifiers. GigaScience 1: 3 .

Wu H, Caffo B, Jaffee HA, Irizarry RA, Feinberg AP. 2010. Redefining CpG islands using hidden Markov models. Biostatistics 11: 499-514.
Xu Y, Wu F, Tan L, Kong L, Xiong L, Deng J, Barbera AJ, Zheng L, Zhang H, Huang S, et al. 2011. Genome-wide regulation of $5 \mathrm{hmC}, 5 \mathrm{mC}$, and gene expression by Tet1 hydroxylase in mouse embryonic stem cells. Mol Cell 42: 451-464.

Yu M, Hon GC, Szulwach KE, Song CX, Zhang L, Kim A, Li X, Dai Q, Shen Y, Park B, et al. 2012. Base-resolution analysis of 5-hydroxymethylcytosine in the mammalian genome. Cell 149: 1368-1380.

Zhang Y, Liu T, Meyer CA, Eeckhoute J, Johnson DS, Bernstein BE, Nusbaum C, Myers RM, Brown M, Li W, et al. 2008. Model-based analysis of ChIPSeq (MACS). Genome Biol 9: R137.

Zhou X, O'Shea EK. 2011. Integrated approaches reveal determinants of genome-wide binding and function of the transcription factor Pho4. Mol Cell 42: 826-836.

Received August 1, 2013; accepted in revised form May 5, 2014. 


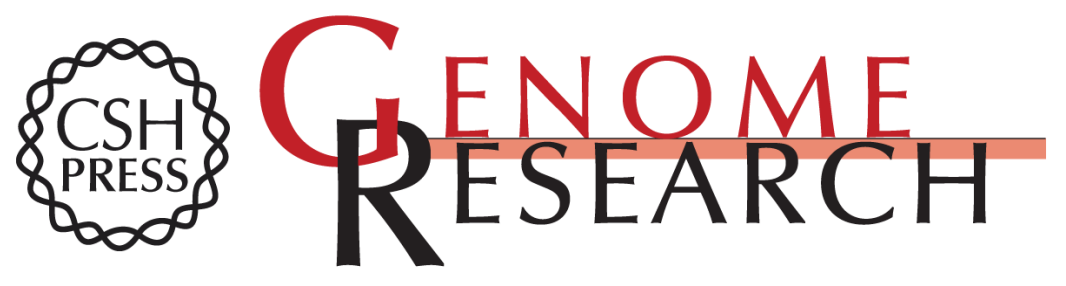

\section{Nucleosome repositioning links DNA (de)methylation and differential CTCF binding during stem cell development}

Vladimir B. Teif, Daria A. Beshnova, Yevhen Vainshtein, et al.

Genome Res. 2014 24: 1285-1295 originally published online May 8, 2014 Access the most recent version at doi:10.1101/gr.164418.113

Supplemental Material

References

Creative

Commons

License

Email Alerting Service
http://genome.cshlp.org/content/suppl/2014/06/05/gr.164418.113.DC1

This article cites 69 articles, 13 of which can be accessed free at: http://genome.cshlp.org/content/24/8/1285.full.html\#ref-list-1

This article is distributed exclusively by Cold Spring Harbor Laboratory Press for the first six months after the full-issue publication date (see

$\mathrm{http}: / /$ genome.cshlp.org/site/misc/terms.xhtml). After six months, it is available under a Creative Commons License (Attribution-NonCommercial 4.0 International), as described at http://creativecommons.org/licenses/by-nc/4.0/.

Receive free email alerts when new articles cite this article - sign up in the box at the top right corner of the article or click here.

\section{Affordable, Accurate Sequencing.}

To subscribe to Genome Research go to:

https://genome.cshlp.org/subscriptions 